\title{
Chemical engineering in the electronics industry: progress towards the rational design of organic semiconductor heterojunctions
}

\author{
Paulette Clancy
}

We review the current status of heterojunction design for combinations of organic semiconductor materials, given its central role in affecting the device performance for electronic devices and solar cell applications. We provide an emphasis on recent progress towards the rational design of heterojunctions that may lead to higher performance of charge separation and mobility. We also play particular attention to the role played by computational approaches and its potential to help define the best choice of materials for solar cell development in the future. We report the current status of the field with respect to such goals.

\section{Address}

School of Chemical and Biomolecular Engineering, Cornell University, Ithaca, NY 14853, United States

Corresponding author: Clancy, Paulette (pqc1@cornell.edu)

Current Opinion in Chemical Engineering 2012, 1:117-122

This review comes from a themed issue on

Nanotechnology

Edited by Hua Chun Zeng

Available online 4th February 2012

2211-3398/\$ - see front matter

(C) 2012 Elsevier Ltd. All rights reserved.

DOI 10.1016/j.coche.2012.01.001

\section{Introduction}

Developments in electronic devices over the past 50 years have revolutionized the way we conduct our daily lives, including the use of organic electronics in cell phones, display technologies and sensors, and ultra-fast processors that have improved the speed of computers in ways unimaginable a generation ago. For example, cell phone subscriptions worldwide passed 5 billion customers in 2010. The electronics industry is a major contributor to the global economy, valued at more than $\$ 300$ billion in 2010 [1].

\section{Chemical engineering in the semiconductor industries}

Chemical Engineers have played a strong role in the development of semiconductor materials and processing. Indeed, the American Institute of Chemical Engineers reports that $\sim 15 \%$ of graduating BS chemical engineers are employed in the electronics industries each year [2].
This is typically the second or third highest percentage destination behind chemicals $(\sim 25 \%)$, similar to fuels (12-20\%), and above foods and pharmaceutical employment. At the PhD level, employment of chemical engineers by the electronics industry is around $20-30 \%$, vying for the most popular destination with chemicals. The attraction of hiring chemical engineers in the electronics industry is clear: Many fabrication processes and materials design issues require a deep understanding of the underlying chemistry, physics and mathematics, and especially of thermodynamic and kinetic processes coupled to chemical reactions and reactor design that are the hallmark of a classically trained chemical engineer. Chemical engineers are trained to understand and apply physical and chemical concepts over extensive orders of magnitude in length-scale (and often time-scale). For example, understanding the atomic-level (sub-nm) details of charge absorption and separation, the multi-nanometer concepts of phase segregation or charge diffusion, and macroscopic (meter-scale) aspects concerning highthroughput processes such as the roll-to-roll processing of organic thin film devices.

The interplay between chemical engineering and nanotechnology/electronics is likely to strengthen in the years ahead. For example, the focus of many modern chemical engineering departments is increasingly at the molecular scale; hence, nanotechnology and molecular-scale processing profitably draw upon the educational training of a chemical engineer. The tradition of chemical engineers in the energy industry is expanding beyond oil and gas industries to new energy opportunities in solar energy, batteries, biomass conversion, and photosyntheticinspired processes. This opens the door to an expanded role for chemical engineers in green energy solutions. Further, many molecular-level aspects concerning the success of organic devices are beyond the capabilities of current experimental techniques and require the assistance of computational methods. Moreover, computational methods not only offer an enhanced analysis of existing material systems, but also offer the exciting prospect of 'materials by design' or computationally led materials discovery, as will be discussed below. A recent article points out that 'computational modeling has long served as a central component of the chemical engineering toolkit' [3] and predicts that 'density functional theory and Molecular Dynamics will one day do for chemical engineering what finite element modeling did for mechanical engineering.' [3] Stapleton writes that computational 
engineering will 'transform' biochemical engineering in the next 25 years. This can also be said for the role of computational engineering on the field of electronics and nanotechnology, and the development of green energy technologies. In the future, chemical engineers are sure to play a strong role, given their inherently systems-based education, on life cycle analysis and issues of broader concern in the renewable energy and sustainability spheres.

\section{Solar energy and the role of the heterojunction}

We focus here on the role of organic semiconductors as potential candidate materials for solar energy production $\left[4^{\bullet}, 5\right]$. Within this domain, we limit our discussion to the heterojunction in solar cells. This (single or multiple) interface between two dissimilar materials is the most critical factor in determining the efficiency of charge separation and transport, as explained below. Organic semiconductors are '2nd generation' photovoltaic (PV) materials. They are a relatively new and not particularly efficient choice for solar cells, with minimal market share. In contrast, Si solar cell technology is well established and current engineering work is mostly focused on cost reduction. Contrasts between organic and inorganic $\mathrm{PV}$ materials are well covered in the literature $\left[6^{\bullet \bullet}\right]$. The efficiency of organic solar cell materials is approaching the $\sim 10 \%$ value for power conversion efficiency that is generally considered the economically viable threshold for widespread deployment. While amorphous silicon $(\sim 12-15 \%)$ and crystalline silicon (25-30\%) remain 'gold standards' for efficiency, approaching the ideal Shockley-Queisser limit ( 33\%) for a single $\mathrm{p}-\mathrm{n}$ junction [7], the competitive 'edge' of all-organic and hybrid organicinorganic solar cells is their inexpensive cost, plentiful raw materials, and opportunity to use solution processing. The key metric for the widespread deployment solar cells is, however, not efficiency but cost per Watt. As of the date of publication, this 'levelized cost of electricity' [8] for solar energy is in the neighborhood of $\$ 0.75 \mathrm{~W}^{-1}$.
When sunlight interacts with a suitable solar cell an exciton (an excited electron-hole pair) is formed which diffuses through the (absorber) material until it reaches a heterojunction formed by the interface between two dissimilar materials (akin to a $\mathrm{p}-\mathrm{n}$ junction). This interface is designed to attract electrons preferentially to one material and holes to the other material. This charge separation leads, ultimately, to the transport of electrons and holes to different electrodes and produces an electric current. If the energy of the exciton decays before it can reach this heterojunction, the charges can recombine and the absorbed energy is lost. There are other mechanisms whereby the energy is lost, including recombination at defects or at charge traps. In fact, the biggest loss mechanism is the loss of 'excess energy' of the photo-excited carrier, that is, energy of the 'hot' electron above the conduction band. This has inspired the development of multi-junction cells, or multi-exciton cells. The exciton diffusion length is short, typically only a few $\mathrm{nm}$ for organic semiconductors. Thus, only excitons relatively near the heterojunction can be expected to charge-separate and contribute to the generation of charge carriers. The basic principles are well described by Nelson $\left[9^{\bullet \bullet}, 10\right]$. Many excellent and recent reviews exist $\left[11^{\bullet}, 12^{\bullet}, 13,14\right]$.

Much of the experimental focus for developing efficient organic solar cells has centered on designing a heterojunction that maximizes charge separation; see Figure 1 for a schematic of interface designs. Planar organic interfaces, such as that in Figure 2, offer the advantage of ease of creation and control over the morphology and distance that excitons have to travel. Very thin films ensure that excitons can diffuse to the interface before the charges in the exciton recombine. While so-called 'bulk' interfaces, featuring dispersed domains of different semiconducting materials, ameliorate the charge recombination problem, the random array of domains provides little control over charge transport. Indeed, it is somewhat surprising that organic PV devices work as well as they do, because the

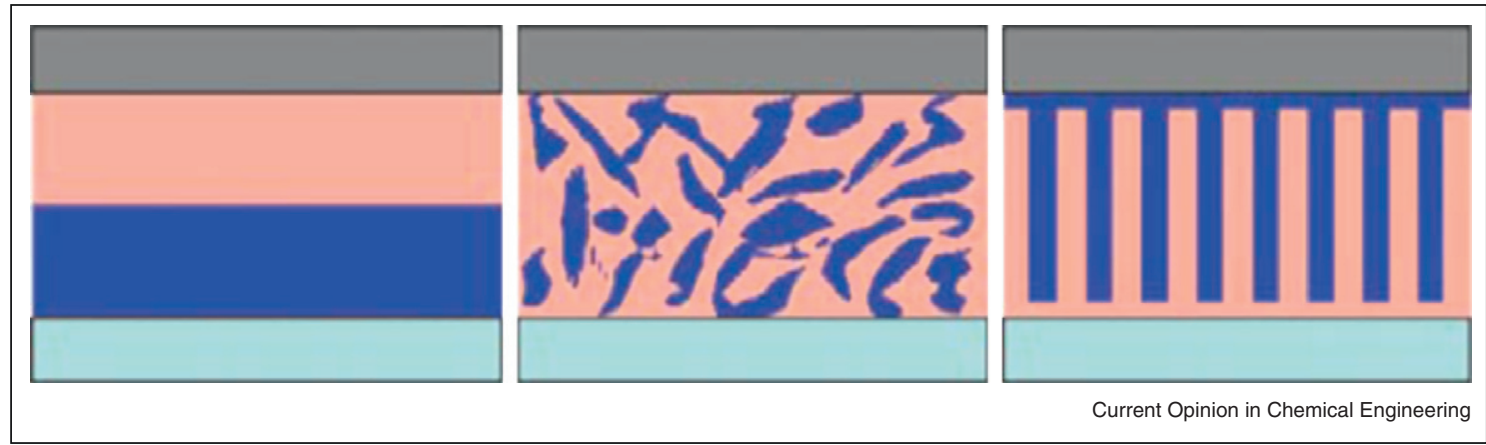

Common heterojunction designs. From left to right: Planar, 'bulk' and ordered heterojunction interfaces. Taken from Mayer AC, Scully SR, Hardin BE, Rowell MW, McGehee MD: Mater Today 2007:10, 11, 28. 
Figure 2

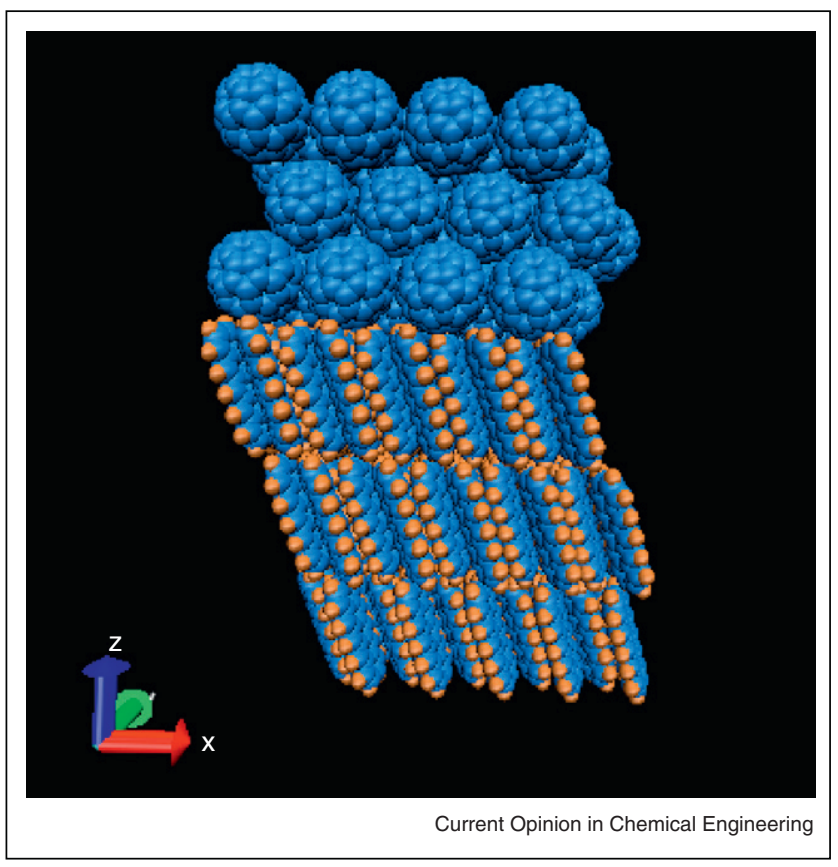

Example of a planar heterojunction showing the abrupt interface between C60 (top) and pentacene (bottom) as described by a snapshot from a Molecular Dynamics simulation.

phase segregation into electron-transporting and holetransporting domains relies on the (fortuitous) formation of bicontinuous structures. Further, efficient charge transport assumes that a continuous path exists to the relevant electrodes. The kinds of materials involved in solar cells range from inorganic nanocrystals in $\pi$-conjugated polymers (see, e.g. [15]) and nanocrystals in polymers [16], to all-organic heterojunctions, such as modified (TIPs) pentacene materials [17], blends of polyphenylvinylene (PPV) and methano-fullerenes [18] and poly(3-hexylthiophene) (P3HT)-fullerene derivatives [19] and solid-state dye-sensitized solar cells [20].

The lure of improved efficiency in charge transport and greater control over the morphology has spurred considerable interest in developing an ordered set of heterojunctions that could lead to 'superhighways' for electrons and holes. Several promising families of candidate materials are already under study. For instance, 'covalent organic framework' materials offer advantages of 'pipelining' electrons and holes in more rigid and more ordered structures with (perhaps) less processing issues [21,22]. Closer to device implementation, Koch and co-workers [23] have used glancing angle deposition to create nanocolumns of interpenetrating morphology. This design gives rise to encouragingly high efficiencies. McGehee et al. have also provided processing routes to ordered organic PV materials in innovative intercalated structures [24,25].
There is a critical need for computational methods to help solve the challenge of their optimal design. The heterojunction is buried within the device and inaccessible to most experimental tools. This defines a unique opportunity for computationally led discoveries, both for better fundamental understanding of the underlying charge transfer processes, and for the prediction of novel materials with better characteristics.

\section{The role for theory: Rational design of heterojunction components}

The early history of material choices for all-organic solar cells was largely a matter of trial and error, given the almost limitless palette of possible molecules. The focus for $p$-type materials was originally centered on the acenes [26], particularly pentacene, whose solar cell efficiencies were in the $1-2 \%$ range, close to amorphous silicon. Thiophenes and thiophene derivatives have attracted attention [27], including now a far larger palette of perylenes, rubrene and more exotic examples [28,29]. Selection of an $n$-type material started with $\mathrm{C}_{60}$, whose electron mobility is close to silicon. The field moved its attention to the solution-processable PCBM (6,6-phenyl-C61-butyric acid methyl ester) and its derivatives [30]. More recently, other nonfullerene molecules, such as parylenes and perylene diimide thin films have dramatically improved electron mobilities to around $1-3 \mathrm{~cm}^{2} /(\mathrm{V} \mathrm{s})$ [31]. In the past few years, there has been a move towards a more rational design of heterojunction materials. This requires that we understand the interaction (and packing) between donor and acceptor molecules and the chemical design of these molecules that ultimately determines both intermolecular interactions and packing. We will explore progress towards rational design of these materials in the remainder of this paper.

Charge transport in organic semiconductors is widely represented as occurring through charges 'hopping' from one molecule to another. Band-bending and other concepts from conventional semiconductors have only limited applicability here. Theories exist to capture this hopping mechanism for charge transport [11 $\left.{ }^{\bullet}, 32,33\right]$, but the estimation of charge mobilities remains limited by the need to find values for the relevant molecular parameters. Charge carrier diffusion is coupled to vibrations in the lattice (both intermolecular and intramolecular). Researchers have interpreted these coupling constants as arising from local electron-vibration site energies, or reorganization energies in Marcus-Hush (electron-transfer) theory [34], and nonlocal electronvibration coupling, the dependence of so-called 'transfer integrals' on the relative orientations of neighboring molecules and their separation. The need to achieve favorable intermolecular orientations for charge transfer leads to a desire for increased order in the material $\left[35^{\circ}\right]$. A 'tutorial review' by Troisi $\left[11^{\circ}\right]$ describes how this small polaron theory interpretation of charge transport in 
molecular crystals has been revised in the light of recent experimental studies which show that band-like transport is possible in highly purified and carefully created samples that were designed to minimize defect concentrations. No temperature exists at which these ultra-pure materials show a 'hopping' transport. Troisi concedes that the language of small polaron transport is so ingrained in our interpretation of studies of small organic semiconductors that discussion of hopping transport is likely to continue long after its applicability has been refined. These results also call to the fore the potentially significant role of defects and grain boundaries to create charge trap states, and the lack of understanding of charge states at heterojunctions. The link between order at the heterojunction and charge mobility is still largely unknown. Verlaak et al. [36] note: '... exact details of the energetic landscape near an organic semiconductor hetero-interface, and its implications for the dynamics of charge dissociation and recombination, remain largely unexplored.'

Computational studies have provided considerable insight to help remediate this situation. There is some convergence towards a strategy for a computational route between modeling a material and predicting its more macroscale mobility: This invariably involves a sequence of computational methods that cover multiple scales from the quantum scale to mesoscale (or macroscale). A typical approach is to use Density Functional Theory (DFT) or other quantum chemical approaches, often in conjunction with Molecular Dynamics (MD), to obtain a geometry optimization of representative motifs of the system, followed by a calculation of transfer integrals and reorganization energies and, finally, a Kinetic Monte Carlo (KMC) simulation using this library of energies as the input parameters [37-40]. Poole-Frenkel plots (mobility versus field strength) have shown that energetic disorder can reduce the mobility by many (at least six) orders of magnitude! [38] This computational protocol is often undertaken in conjunction with Wide Angle X-ray Scattering (WAXS) and/or NMR experimentation $\left[41^{\circ}, 42^{\bullet}\right]$.

Studies of the effect of defects and grain boundaries are less common: Rivnay et al. adopt a quantitative measure of order through a paracrystallinity parameter, $g$ [43 $\left.{ }^{\circ}\right]$. Disorder in PBTTT (poly[2,5-bis(3-alkylthiophen-2yl)thieno(3,2-b)thiophene]), for example, has a paracrystallinity index of $7 \%$ ( $10 \%$ is considered amorphous). Gap states are more numerous with increasing $g$ (disorder). Troisi $\left[11^{\bullet}\right]$ quantifies the lifetime of trap states to be on the order of $0.1-1 \mathrm{ps}$ for hole trap states $0.3 \mathrm{eV}$ above the valence band edge of pentacene and suggests that slow spectroscopies may be 'blind' to the traps' existence and more likely to probe 'bulk' charge carriers' states. Vehoff

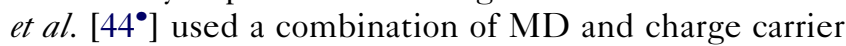
dynamics simulations to investigate the dependence of charge transport on morphology and the dimensionality of the percolation network. Hole mobilities were generally over-estimated by 3-4 orders of magnitude (except for rubrene) and assumed to be due to the neglect of grain boundaries and defects. It is thus reasonable to conclude that charge transport is defect-limited.

For future effective heterojunctions, the experimental challenges will involve managing a complex set of variables including processing and environmental conditions that affect the structure of the interface and hence the device operability. Each of these steps involves uncertainties and stochastic outcomes. From a computational viewpoint, the challenges are different, but equally difficult. For computation, intermolecular and intramolecular interactions govern the eventual outcome, but expressing these forces is an ongoing challenge [45]. A second major challenge is to leverage and scale this knowledge to move from the electronic structure or molecular level simulations to experimentally relevant length-scale and timescale. Few, if any, packages seamlessly pass information from one simulation package (say, electronic structure to Molecular Dynamics to lattice-based Kinetic Monte Carlo simulations).

The drive to use computational approaches to rationally design organic semiconductors has some momentum. For example, Bao and Aspuru-Guzik used Density Functional Theory screening and Molecular Mechanics to select a particularly high-mobility molecule [46]. Yong and Zhang use a combination of DFT and time-dependent DFT to select candidate electron-donor molecules [47]. Forrest provided some rules to achieve efficiencies above $1 \%$ [48], quoting an upper bound for the efficiencies of small molecule organic solar cells of about $20 \%$, which is above the threshold for economic viability. In the search for design rules, it is just as important to define the boundaries at which an approach becomes ineffective; see for example, Refs. [48-51].

Finally, despite the experimental complexities involved, the future of solar cells will surely lie in the design of multi-junction cells with the lure of exceeding the Shockley-Queisser limit. PV devices with cascaded energy gaps more efficiently harvest the broad-solar spectrum and thereby ameliorate the biggest loss mechanism. Recent reviews already exist for tandem cells, focusing on theoretical considerations [52], while organic multi-junction devices are starting to be prepared $[53,54]$.

\section{Summary}

Computational efforts to help guide the selection of candidate materials and the design of heterojunctions for electronic devices appear to be at the onset of some potentially important breakthroughs for small molecule organic devices. We have moved beyond the use of computational assistance to simply explain experimental results and are beginning to uncover rules to help guide experimental choices of molecules. Rational design of 
high-performing materials appears to be an achievable goal.

A suitable computational toolkit exists to provide vital information that we pass from one length-scale and timescale to another. More effort is needed to study the effects of disorder, defects and grain boundaries on mobility. Simulation could also be profitably used to optimize processing methods that minimize the creation of charge traps or suggest materials that finesse their creation. The next major hurdle will be to tackle the reverse design of materials in which we select the material properties and then find materials whose design fulfills these criteria.

\section{Acknowledgements}

My thanks to my colleague, Tobias Hanrath, for carefully reading and improving the manuscript. This publication is based on work supported in part by Award No. KUS-C1-018-02, made by King Abdullah University of Science and Technology (KAUST).

\section{References and recommended reading}

Papers of particular interest, published within the period of review, have been highlighted as:

- of special interest

$\bullet$ of outstanding interest

1. See http://www.electroiq.com/articles/sst/2011/01/the-forecastfor-2011-back-to-reality.html; 2011 (accessed November 15).

2. http://www.aiche.org/Students/Careers/placementsurvey.aspx (accessed 1 December 2011).

3. Westmoreland PR: Chemical Engineering in the Next 25 Years Chem. Eng. Progr. 2008, 104:30-41.

4. DOE BES Report (http://science.energy.gov/bes/news-and-

- resources/reports/abstracts/\#SEU)

This provides a prescient perspective on future PV development.

5. Wolden CA, Kurtin J, Baxter JB, Repins I, Shaheen SE, Torvik JT, Rockett AA, Fthenakis VM, Aydil ES: Photovoltaic manufacturing: present status, future prospects, and research needs. J Vacuum Sci Technol A: Vacuum Surf Films 2011, 29:030801.

6. Gregg B, Hanna M: Comparing organic to inorganic

- photovoltaic cells: theory, experiment and simulations. $J$ App / Phys 2003, 93:3605-3614.

Discusses differences in photogeneration of charge carriers in organics vs. inorganics.

7. Shockley W, Queisser HJ: Detailed balance limit of efficiency of p-n junction solar cells. J Appl Phys 1961, 32:510-519.

8. See the U.S. Energy Information Administration Explanation of This Term at http://38.96.246.204/oiaf/aeo/electricity_generation.html (accessed 20 November 2011)

9. Nelson J: The Physics of Solar Cells. Imperial College Press; 2003.

ISBN: 1860943497

This clearly written and short book is an excellent way for newcomers to the field to understand the basic fundamentals of solar cells.

10. Nelson J, Kwiatkowski JJ, Kirkpatrick J, Frost JM: Modeling of charge transport in organic photovoltaic materials. Acc Chem Res 2009, 42:1768-1778.

11. Troisi $\mathrm{A}$ : Charge transport in high mobility molecular

- semiconductors: classical models and new theories. Chem Soc Rev 2011, 40:2347-2358.

Thought-provoking article summarizing recent experimental research that has clarified the representation of charge transport mechanisms and hence revised theoretical descriptions of these processes. Reviews small polaron theory, the transition from band-like to hopping transport and the studies of ultra-pure pentacene and rubrene.
12. Coropceanu V, Cornil J, da Silva Filho DA, Olivier Y, Silbey R,

- Brédas J-L: Charge transport in organic semiconductors. Chem Rev 2007, 107:926-952.

An excellent review of the fundamentals of charge transport that is required reading for students of the area.

13. Puschnig $P$, Nabok D, Ambrosch-Draxl C: In Toward an Ab-Initio Description of Organic Thin Film Growth, in Interface-Controlled Organic Thin Films,. Edited by Rubahn H-G et al.: Berlin: SpringerVerlag; 2009. doi:10.1007/978-3-540-9530-4.

14. Clancy P: Molecular simulation studies of thin film growth of small molecule organic semiconductor materials. Chem Mater 2011, 23:522-543

15. Gunes S, Neugebauer H, Sariciftci NS: Conjugated polymerbased organic solar cells. Chem Rev 2007, 107:1324-1338.

16. Liu J, Tanaka T, Sivula K, Alivisatos AP, Fréchet JMJ: Employing end-functional polythiophene to control the morphology of nanocrystal-polymer composites in hybrid solar cells. J Am Chem Soc 2004, 126:6550-6551.

17. Lloyd MT, Mayer AC, Tayi AS, Bowen AM, Kasen TG, Herman DJ, Mourey DA, Anthony JE, Malliaras GG: Photovoltaic cells from a soluble pentacene derivative. Org Electron 2006, 7:243-248.

18. Brabec CJ, Padinger F, Hummelen JC, Janssen RAJ, Sariciftci NS: Realization of large area flexible fullerene-conjugated polymer photocells: a route to plastic solar cells. Synth Mater 1999, 102:861-864.

19. Krebs FC: Fabrication and processing of polymer solar cells: a review of printing and coating techniques. Solar Energy Mater Solar Cells 2009, 93:394-412.

20. Grätzel M: Dye-sensitized solar cells. Rev Photochem Photobiol C 2004, 4:145-153.

21. Wan S, Gandara F, Asano A, Furukawa H, Saeki A, Dey SK, Liao L, Ambrolgio MW, Botross YY, Duan $X$ et al.: Covalent organic frameworks with high charge carrier mobility. Chem Mater 2011, 23:4094-4097.

22. Spitler EL, Dichtel W: Lewis acid-catalysed formation of twodimensional phthalocyanine covalent organic frameworks. Nat Chem 2010, 2:672-677.

23. Yu S, Klimm C, Schafer P, Rabe JP, Rech B, Koch N: Organic photovoltaic cells with interdigitated structures based on pentacene nanocolumn arrays. Org Electron 2011, 12:2180-2184.

24. Cates NC, Gysel R, Beiley Z, Miller CE, Toney MF, Heeney M, McCulloch I, McGehee MD: Tuning the properties of polymer bulk heterojunction solar cells by adjusting fullerene size to control intercalation. Nano Lett 2009, 9:4153-4157.

25. Mayer AC, Toney MF, Scully SR, Rivnay J, Brabec CJ, Scharber M, Koppe M, Heeney M, McCulloch I, McGehee MD: Bimolecular crystals of fullerenes in conjugated polymers and the implications of molecular mixing for solar cells. Adv Funct Mater 2009, 19:1173-1179.

26. Anthony JE: Functionalized acene and heteroacenes for organic electronics. Chem Rev 2006, 106:5028-5048.

27. Lloyd MT, Anthony JE, Malliaras GG: Photovoltaics from soluble small molecules. Mater Today 2007, 10:34-41.

28. Scharber MC, Mühlbacher D, Koppe M, Denk P, Waldauf C, Heeger AJ, Brabec CJ: Design rules for donors in bulkheterojunction solar cells - towards $10 \%$ energy-conversion efficiency. Adv Mater 2006, 18:789-794.

29. Venkataraman D, Yurt S, Venkatraman BH, Gavvalapalli N: Role of molecular architecture in organic photovoltaic cells. J Phys Chem Lett 2010, 1:947-958.

30. Wang HH, He Y, Li Y, Su H: Photophysical and electronic properties of five PCBM-like C60 derivatives: spectral and quantum chemicals. J. Chem. Phys. A 2012, 116:255-262.

31. Fabiano S, Wang $H$, Piliego $C$, Jaye $C$, Fischer DA, Chen Z, Pignataro B, Facchetti A, Loo Y-L, Loi MA: Supramolecular order of solution-processed perylenediimide thin films: highperformance small-channel n-type organic transistors. Adv Funct Mater 2011. (Online October 2011). 
32. Noriega $\mathrm{R}$, Salleo A: Charge transport theories in organic semiconductors. In Organic Electronics II: More Materials and Applications. Edited by Klauk H. Wiley VCH; 2011:67-101.

33. Ortmann F, Bechstedt F, Hannewald K: Charge transport in organic crystals: interplay of band structure, hopping and electron-phonon scattering. N J Phys 2010, 12:023011.

34. R.A. Marcus's papers on electron transfer began in 1956 with a paper entitled 'On the Theory of Oxidation-Reduction Reactions Involving Electron Transfer. I'. J Chem Phys 1956, 24:966. His work on outer sphere electron transfer was extended to inner-sphere electron transfer by Hush NS: Adiabatic theory of outer sphere electron-transfer reactions in solution. Trans. Faraday Soc. 1960:57, 557-580.

35. Sánchez-Carrera RS, Paramonov P, Day GM, Coropceanu V,

- Brédas J-L: the Interaction of charge carriers with lattice vibrations in oligoacene crystals from naphthalene to pentacene. J Am Chem Soc 2010, 132:14437-14446.

Provides an example of recent progress on our understanding of nonlocal effects on the acenes.

36. Verlaak S, Beljonne D, Cheyns D, Rolin C, Linares M, Castet F, Cornil J, Heremans P: Electronic structure and geminate pair energetics at organic-organic interfaces: the case of pentacene/C60 heterojunctions. Adv Funct Mater 2009, 19:3809-3814

37. Li Y, Lagowski JB: Charge carrier mobility in conjugated organic polymers - case studies using multi-step computational approach. Polymer 2011, 52:4841-4850.

38. Rühle V, Lukyanov A, May F, Schrader M, Vehoff T, Kirkpatrick J, Baumeier B, Andrienko D: Microscopic simulations of charge transport in disordered organic semiconductors. J Chem Theory Comput 2011, 7:3335-3345.

39. Athanasopoulos S, Emelianova EV, Walker AB, Beljonne D: Exciton diffusion in energetically disordered organic materials. Phys Rev B 2009, 80:195209.

40. Nelson J, Kwiatkowski JJ, Kirkpatrick J, Frost JM: Modeling charge transport in organic photovoltaic materials. Acc Chem Res 2009, 42:1768-1778.

41. Marcon V, Breiby DW, Pisula W, Dahl J, Kirkpatrick J,

- Patwardhan S, Grozema F, Andrienko D: Understanding structure-mobility relations for perylene tetracarboxydiimide derivates. J Am Chem Soc 2009, 131:11426-11432.

This paper shows the power of combining WAXS with MD and NMR for discotic mesophases.

42. Papadopoulos TA, Muccioli L, Athanasopoulos S, Walker AB,

- Zannoni C, Beljonne D: Does supramolecular ordering influence exciton transport in conjugated systems? Insight from atomistic simulations. Chem Sci 2011, 2:1025.

WAXS and MD were used to predict morphology, finding that a decrease in order does not alter the exciton diffusion length, Ld. MD and KMC results for exciton diffusion showed that the diffusion length was not affected by reduced order parameters with increasing temperature. There was a cancelling effect between the excitonic coupling and an increase of spectral overlap.

43. Rivnay J, Noriega R, Northrup JE, Kline RJ, Tomey MF, Salleo A:

- Structural origin of gap states in semicrystalline polymers and the implications for charge transport. Phys Rev B 2011, 83:121306R

Provides a metric for disorder and links this to gap states in the system Article provides a helpful introduction to charge transport for newcomers to the field.

44. Vehoff T, Baumeier B, Troisi A, Andrienko D: Charge transport in

- organic crystals: role of disorder and topological connectivity. $J$ Am Chem Soc 2011, 132:11702-11708.

This study provides some quantification to the effect of defects on transport.

45. Morena M, Casalegn M, Raos G, Meille SV, Po R: Molecular modeling of crystalline alkylthiophene oligomers and polymers. J Phys Chem B 2010, 114:1591-1602.

46. Sokolov AN, Atahan-Evrenk S, Mondal R, Akkerman HB, SanchezCarrera RS, Granados-Focil S, Schrier K, Mannsfeld SCB, Zoombelt AP, Bao Z, Aspuru-Guzik A: From computational discovery to experimental characterization of a high hole mobility organic crystal. Nat Commun 2011, 2:437.

47. Yong $\mathrm{X}$, Zhang J: A rational design strategy for donors in organic solar cells: the conjugated planar molecules possessing anisotropic multibranches and intramolecular charge transfer. J Mater Chem 2011, 21:11159-11166.

48. Forrest SR: The limits to organic photovoltaic cell efficiency. MRS Bull 2005, 30:28-32.

49. Misra M, Andrienko D, Baumeier B, Faulon J-L, von Lilienfeld OA: Toward quantitative structure-property relationships for charge transfer rates of polycyclic aromatic hydrocarbons. Chem Theory Comput 2011, 7:2549-2555.

50. Heremans $P$, Cheyns D, Rand BP: Strategies for increasing the efficiency of heterojunction organic solar cells: material selection and device architecture. Acc Chem Res 2009, 42:1740-1747.

51. Yang F, Forrest SR: Photocurrent generation in nanostructured organic solar cells. ACS Nano 2008, 2:1022-1032.

52. Ameri T, Dennier G, Lungenschmied C, Brabec CJ: Organic tandem solar cells: a review. Energy Environ Sci 2009, 2:347363.

53. Kim JY et al.: Efficient tandem polymer solar cells fabricated by all-solution processing. Science 2007, 317:222-225.

54. Hadipour A, de Boer B, Blom PWM: Organic tandem and multijunction solar cells. Adv Funct Mater 2008, 18:169-181. 